\title{
Parteien: Kritik als Motor der Erneuerung
}

\section{Siri, Jasmin: Parteien. Zur Soziologie einer politischen Form, VS Verlag für Sozialwissenschaften, Wiesbaden 2012, 282 Seiten, $€$ 34,95.}

Jasmin Siri ergänzt mit ihrer Arbeit über Parteien als Organisationen die Parteienforschung um eine explizit soziologische Perspektive. Dazu behandelt sie im ersten Teil die „Krise der Parteien“, um sich dann, aus der Funktionalität der Krise heraus, im zweiten Teil dem „Formenwandel politischer Organisierung“ zuzuwenden. Sie erklärt, warum es ohne Parteien nicht geht.

Siri referiert nicht nur die Klassiker Wladimir I. Lenin, Rosa Luxemburg, Moissei Ostrogorski, Robert Michels, Max Weber und Carl Schmitt, sondern interpretiert deren Ergebnisse aus ihrem zeitlichen und theoretischen Zusammenhang. Nach der synchronen Betrachtung der geistesgeschichtlichen Entwicklung fasst sie die aktuelle Parteienkritik zusammen und blickt dann auf die Wirkung im Formenwandel. Ihre diachrone Dekonstruktion der Krisendiagnose folgt der wiederkehrenden Frage: Was ist wirklich neu? An den Klagen über Argumentationsverlust, zu viel und zu wenig Heterogenität, Führungsdefizite, zu geringe Erneuerung der Eliten, Degradierung der Wähler als Stimmvieh und Selbstalimentation der Parteien zeigt sie, dass die Konsequenzen der paradoxen Anforderung nur selten berücksichtigt werden. Weil Parteien als Teil das Ganze repräsentieren sollen, können sie für zu geringe Beteiligung ebenso wie für unzureichende Entscheidungen kritisiert werden. Gebündelt wird die Kritik in den Anforderungen an die ausführenden Personen. „Politiker scheinen also nicht nur die Aufgabe zu haben, Entscheidungen für eine Gruppe von Menschen zu fassen. Sie haben auch die Aufgabe, ihr Vermögen, richtige Entscheidungen zu treffen, durch authentisches Verhalten habituell zu reproduzieren und interaktional wie medial zu symbolisieren" (S. 123). Sie sollen in beschleunigter Berufspolitik massenmedial vermitteln und dabei trotz der ,zerstörerischen Macht des grauen Mittelmaßes“ in Orts- und Kreisverbänden anwesend sein. Obwohl gilt, „Politiker dürfen nicht gefallen wollen“ (S. 126 f.), sollen sie doch emotional binden. Die Grundlage dieser Klagen lokalisiert Siri in der Enttäuschung darüber, dass der kollektive Wille einer Versammlung nicht aus sich selbst hervorgeht, sondern eben erst durch eine Parteitagsregie hergestellt und durch Akklamation symbolisiert werden muss (S. 117).

Zusammenfassend stellt sie fest, dass sich Themen und Kritik der Krisenkommunikation kaum verändern. Sie knüpfen an brandaktuelle politische Ereignisse an, bleiben aber für ihre eigenen Historizität eigentümlich blind. In der Diskursanalyse zeigt Siri, dass sie die Aufgabe haben, normative Kommunikation in die Politik der Gesellschaft einzuspeisen und so gegen die Eigendynamik einer differenzierten Gesellschaft als Substanz demokratischer Entscheidung - Identität von Volk und Gesellschaft zu symbolisieren (S. 138) und damit legitimatorisch zu wirken. Empirisch zeigt sich dies auch darin, dass Diktaturen keine „Krise der Partei(en)“ herstellen können“ (S. 142).

Aus der Tatsache, dass Parteien trotz permanenter Kritik weder untergehen noch durch subpolitische Akteure ersetzt werden, schließt Siri, dass moderne Parteien und deren Mitgliedschaft durch den Hinweis auf schwindende Bindung, sinkende Mitgliederzahlen und ideologische Auflösungserscheinungen nicht ausreichend soziologisch beschrieben werden.

Nach der Soziologie der Krise wendet sich Siri im zweiten Teil dem Formwandel politischer Organisierung zu. Mit den Fragen: (1) „Anhand welcher Programme ... versuchen die Parteien durch organisationalen Wandel mit der gesellschaftlichen Entwicklung umzuge- 
hen?; (2) wie gehen Mitglieder damit um, dass eine Parteimitgliedschaft „von der Wiege bis zur Bahre" eine für die moderne Biografie unwahrscheinliche Erzählung darstellt?; (3) in welchem Verhältnis stehen politische Beratung und Beratene?" (S. 151) verbindet Siri empirische Forschung und Theoriedebatten der Soziologie. Sie nimmt mit Wahlkampf und Kampagnen, Programmen, Erzählung der Mitgliedschaft und politischer Beratung organisatorische Gegenwarten in den Blick, die jeweils eigenlogisch funktionieren und miteinander oft weniger zu schaffen haben, als mediale und wissenschaftliche Beobachter meinen.

Jasmin Siri leistet mit dem Perspektivenwechsel von der „Fiktion einer identen ParteienPerson“ hin zu Parteien als paradoxen Organisationen einen originären wissenschaftlichen Beitrag. Aus der Widersprüchlichkeit der Anforderungen durch Legitimation/Partizipation und Effektivität/handlungsbefähigende Geschlossenheit kann sie ableiten, dass Parteienkritik zwar ihren Ursprung in den unerfüllbaren Erwartungen identitärer Demokratie hat, aber als Motor permanent zu Erneuerung und Reform herausfordert. „Die paradoxe Wirkung der Kritik an den Parteien besteht in ihrer Funktionalität für die Reproduktion der Organisationen und des politischen Systems. Die Kritik ermöglicht das Präsenthalten demokratischer Ideale, die Krisendiagnostik das Anstoßen von Reformen“" (S. 254).

Da es kaum Arbeiten über Wahlgesetze und die unterschiedlichen Satzungen der Parteien gibt, öffnet sich mit der Aufmerksamkeit für Organisation ein neues Forschungsfeld. Mit dem Blick auf die Aufgaben von Parteien und deren Gemeinsamkeiten wird es möglich, über Einzelstudien zu CDU, SPD, FDP, Grünen, Linken und Piraten hinaus zu untersuchen, unter welchen Wahlgesetzen und mit welchen Satzungen sich die widersprüchlichen Anforderungen wenigstens ansatzmäßig vereinbaren lassen. Den Praktikern in den Parteien dürfte der Hinweis auf die strukturellen Brüche helfen, die widersprüchlichen Erwartungen als nicht nur individuelles Problem zu begreifen. Beratern in und außerhalb der Parteien könnten die systematische Darstellung des nicht zu unterschätzenden Wandels der Parteiorganisationen durch immer mehr Kontakte mit den Massenmedien und wachsenden Bedarf an Beratungskommunikation nützen. Die nicht einfache Lektüre des Textes lohnt sich und lädt den interessierten Leser ein zu prüfen, welche Bedingungen, Erwartungen und Aporien in die eigenen Analysen einfließen.

Ingrid Reichart-Dreyer

\section{SPD: Personen und Programme am Ende der Ära als Volkspartei}

Reinhardt, Max: Aufstieg und Krise der SPD. Flügel und Repräsentanten einer pluralistischen Volkspartei, Nomos Verlagsgesellschaft, Baden-Baden 2011, 628 Seiten, € 99,-.

Sachs, Matthias: Sozialdemokratie im Wandel. Programmatische Neustrukturierungen im europäischen Vergleich, VS Verlag für Sozialwissenschaften, Wiesbaden 2011, 381 Seiten, € 39,95.

Die gute alte SPD, die Sozialdemokratie als Volks- und Mitgliederpartei, auch als kulturelles Milieu und als soziale Bewegung wird - 150 Jahre nach ihrer Gründung - immer mehr zu einem geschichtlichen Phänomen, versinkt gleichsam im Horizont der Historie. Je mehr jedoch diese Partei zur Vergangenheit wird, desto intensiver befassen sich Historiker, Politikund Sozialwissenschaftler mit ihr, wird gefragt, ob die Solidargemeinschaft, die Volkspartei, 